strain under equivalent cultural conditions about $15 \mathrm{~h}$ after transfer to sporulation medium ${ }^{5}$.

The procedures were done twice with both vegetative and 6-day sporulated cultures of Saccharomyces oleaceus Santa Maria and $S$. mellis (Fabian and Quinet) Lodder and Kreger-van Rij. Proline was not detected in the vegetative cells of either yeast; but it was prominent among the amino-acids in sporulated cells. However, no proline was found in four experiments using vegetative and sporulated cells of Schizosaccharomyces pombe Lindner, nor in an experiment with Torulopsis famata (Harrison) Lodder.

Changes in amino-acid content after transfer of 6-day sporulated cells of $S$. cerevisiae to fresh growth medium were also followed. In about $4 \mathrm{~h}$ the spores were distinctly swollen. A day later most had germinated, and growth was in progress. Whereas most amino-acids gave evidence of increase during two days in growth medium, proline diminished to $200 \mu \mathrm{g} / \mathrm{g}$.

It would have been preferable to use for these experiments large volumes of sporulation medium, taking successive samples during six days, instead of running each chromatogram with an individual $100-\mathrm{ml}$. culture. But as sporulation was poor in large sporulation cultures, the less-accurate method had to be used, and this may account in part for the fluctuations in amount of glutamic acid and glutathione indicated by successive determinations. But as each point is based on at least four, and in many cases ten, experiments it is possible that marked changes occur in the content of these substances during active sporogenesis.

We thank the National Research Council of Canada for the award to one of us (C. R.) of a postdoctorate fellowship.

C. Ramirez

J. J. MILLER

Department of Biology

McMaster University, Hamilton, Ontario.

${ }^{1}$ Ljungdahl, L., and Sandegren, L., Acta Chem. Scand., 4, 1150 (1950).

"Lindan, O., and Work, E., Biochem. J., 48, 337 (1951).

${ }^{3}$ Taylor, E. S., J. Gen. Microbiol., 3, 211 (1949).

${ }^{4}$ Halvorson, H. O., and Spiegelman, S., J. Bact., 65, 601 (1953).

${ }^{5}$ Pontefract, R. D., and Miller, J. J., Canad. J. Microbiol., 8, 573 (1962).

${ }^{6}$ Connell, G. E., Dixon, G. H., and Hanes, C. S., Canad. J. Biochem. and Physiol., 33, 416 (1955).

' Hanes, C. S., Canad. J. Biochem. and Physiol., 39, 119 (1961).

${ }^{*}$ Wade, E. H. M., Matheson, A. T., and Hanes, C. S., Canad. J. Biochem. and Physiol., 39, 141 (1961).

- Hanes, C. S., Harris, C. K., Moscarello, M. A., and Tigane, L., Canad. $J$. Biochem. and Physiol., 39, 163 (1961).

\section{Stimulation of Sporulation in Penicillium by Anhydroglucose}

IN submerged shaken cultivation in a synthetic medium containing potassium nitrate, potassium dihydrogen phosphate, and magnesium sulphate, trace elements and pure (analytical reagent) glucose, Penicillium griseofulvum Dierckx (Strain $A R L$. 375) grows vegetatively but does not sporulate even when it has used up all the available nitrogen. Abundant sporulation at a relatively early, exponential, stage of growth was observed when pure glucose was replaced by a crude commercial glucose derived from maize starch by acid hydrolysis. ${ }^{1}$ Crude glucose was also found to stimulate submerged sporulation in 5 other species of Penicillium out of a further 21 species which were tested. The nature of the sporing factors in crude glucose was therefore investigated.

It was found that the sporing stimulus depended on the simultaneous presence of two substances, of which the first proved to be calcium $\left(\mathrm{Ca}^{++}\right)$, occurring in amounts suffi. cient to give a level of approximately $5 \times 10^{-4} \mathrm{M}$ in a medium containing $5 \mathrm{~g} / \mathrm{l}$. crude glucose. Calcium was already known to induce submerged sporulation of Penicillium griseofulvum, but only at levels above $5 \times$ $10^{-3} \mathrm{M}$, and then only at a late, post-exponential stage of growth when the nitrogen in the medium is either exhaus. ted or nearing exhaustion. The amount of calcium present in the crude glucose added to our standard medium doos not, however, normally induce sporulation, except in the presence of a second substance occurring in crude glucose. This was found to be a neutral organic heat-stable compound which appeared to be an acid reversion product of glucose. In fact variable amounts of a similarly active substance were found to be produced by autoclaving pure glucose with acid. The substance was separated from crude glucose by adsorption on charcoal and elution with ethanol, and the active fraction was identified as a 1,6anhydroglucose by preparative paper chromatography. When this fraction, together with $1 \times 10^{-4} \mathrm{M} \mathrm{Ca}^{++}$, was added to a submerged culture of $P$. griseofulvum in a medium containing pure glucose, abundant sporulation was induced early in the exponential phase of growth, although neither $\mathrm{Ca}^{++}$nor the anhydroglucose fraction produced this sporing effect alone.

The nature of the organic sporing factor was confirmed by synthesizing 1,6-anhydro- $\beta$-D-glucopyranose by alkali treatment of $\beta$-phenylglucoside ${ }^{2}$. This substance gave the characteristic induction of sporulation in $P$. griseofulvum when added, with $1 \times 10^{-4} \mathrm{M} \mathrm{Ca}^{++}$, to a culture growing in medium containing pure glucose. Maximal sporulation occurred when the anhydroglucose concentration was between $2 \times 10^{-4}$ and $6 \times 10^{-4} \mathrm{M}$ and was not increased at higher concentrations. No sporulation occurred in the absence of added $\mathrm{Ca}^{++}$even with very high concentrations of anhydroglueose in the usual growing medium. When the potassium nitrate was replaced by ammonium succinate, however, very high concentrations of anhydroglucose did induce (late) sporing witbout added $\mathrm{Ca}^{++}$. This does not seem to be due to traces of $\mathrm{Ca}^{++}$already present, and it indicates, as do certain accompanying morphological changes, that anhydroglucose may have specific biological effects apart from its interaction with $\mathrm{Ca}^{++}$. A mixture of the pyranose and furanose forms of 1,6-anhydro-D-glucose, produced by the pyrolytic distillation of starch $^{3}$, proved to have the same sporulation-inducing effect as the pure pyranose form, when tested (with $\mathrm{Ca}^{++}$) in the same conditions. Smaller but significant inductions of sporulation were also given in the presence of $\mathrm{Ca}^{++}$by 1,6 -anhydro- $\beta-\mathrm{D}$ galactopyranose and by 1,6-anhydro- $\alpha$-D-galactofuranose.

These observations show that the stimulus to sporulation of $P$. griseofulvum (and probably of other species of Penicillium) caused by crude glucose is due to the presence of anhydroglucose and traces of $\mathrm{Ca}^{++}$. Further confirmation is provided by the absence of any sporing effect from crude glucose produced by enzyme hydrolysis instead of by acid hydrolysis of starch. Studies of the effects of anhydroglucose and related compounds on metabolism and sporulation of fungi are now in progress. The ability of 1,6-anhydro- $\beta$-D-glucopyranose to increase so powerfully the spore-inductive effect of $\mathrm{Ca}^{++}$provides another example of a biological effect elicited by carbohydrate reversion products ${ }^{4,5}$. Interactions between $\mathrm{Ca}^{++}$and hexose phosphates have been observed in the stimulation of perithecia formation in Chaetomium ${ }^{6}$.
J. J. Armstrong
D. J. F. ENGLAND
A. G. Monton J. A. WeBB

Imperial Chemical Industries, Ltd.

Pharmaceuticals Division,

Akers Research Laboratories, Welwyn, Herts.

${ }^{1}$ Morton, A. G., England, D. J. F., and Towler, D. A., Trans. Brit, Myeol. Soc., 41, 39 (1958).

${ }^{2}$ Montgomery, E. M., Richtmeyer, N. K., and Hudson, C. S., J. Amer. Chem. Soc., 65, 3 (1943).

${ }^{3}$ Dimler, R. J., Davis, H. A., and Hilbert, G. E., J. Amer. Chem. Soc., 68. 1377 (1946)

- Mandels, M., and Reese, E. T., Biochem. Biophys. Res. Comm, 1, 338 (1959).

'Slonimski, P., Defaye, J., Asselineau, J., and Lederer, E., C.R. Acad. Sci.. Paris, 249, 192 (1959).

- Buston, FI. W., Jabbar, A., and Etheridge, D. E., J. Gen. Microbiol., 8, 302 (1953). 